\title{
I. Concepts et méthodologie
}

\section{Claire Dubus}

\section{(2) OpenEdition}

Journals

Édition électronique

URL : https://journals.openedition.org/eastafrica/555

DOI : $10.4000 /$ eastafrica.555

ISSN : 2790-1076

Éditeur

IFRA - Institut Français de Recherche en Afrique

Édition imprimée

Date de publication : 1 avril 2010

Pagination : 19-42

ISSN : 2071-7245

\section{Référence électronique}

Claire Dubus, «I. Concepts et méthodologie », Les Cahiers d'Afrique de l'Est / The East African Review [En ligne], 43 | 2010, mis en ligne le 07 mai 2019, consulté le 09 décembre 2021. URL : http:// journals.openedition.org/eastafrica/555 ; DOI : https://doi.org/10.4000/eastafrica.555

Ce document a été généré automatiquement le 9 décembre 2021

Les Cahiers d'Afrique de l'Est / The East African Review 


\title{
I. Concepts et méthodologie
}

\author{
Claire Dubus
}

\subsection{Sur la musique en général}

\subsubsection{Définition basique de l'objet musique}

1 La musique est un objet immatériel; les définitions sont nombreuses, et il n'est pas question ici de poser une réflexion sur la nature de la musique en tant que telle. On tiendra pour acquis la définition de base de l'objet « musique » comme un ensemble de sons et de silences élaboré consciemment par l'être humain dans un but autre que celui de la communication directe. La musique est couramment désignée comme un des principaux arts c'est-à-dire une "des activités créatrices visant à produire une expression esthétique » (Knafou, 2003 : 89), et sa définition se partage entre deux approches. La première, intrinsèque ou immanente, pose le principe que la musique préexiste à l'œuvre musicale. Pour la seconde, fonctionnelle, il n'existe de musique que quand il y a effectivement combinaison de sons et de silences, et que cette combinaison est reçue par un auditoire. Elle se situe dans une dialectique de la création, mais aussi de la diffusion et de la réception.

On se tiendra à cette approche fonctionnelle de la musique, et à cette définition de base, en nuançant d'emblée la dimension artistique de la musique. L'utilisation qui est faite de la musique dans l'ensemble des sociétés, contemporaines ou non, traduit en effet une dimension a priori plus large que le simple esthétisme. La musique, support et sujet de loisirs, de divertissements, mais aussi de rites sociaux et de transactions commerciales, doit être considérée comme un objet dont le prisme d'observation doit être élargi au maximum.

\subsubsection{Premières observations sur la musique}

3 D'emblée une définition satisfaisante et complète de la musique semble difficile à atteindre. On peut donc adopter une démarche en creux : analyser les contours de l'objet musique, voir quelles sont ses fonctions et les utilisations qui en sont faites peut 
être un moyen de mieux cerner cet objet immatériel; définir aussi la musique par rapport à ce qu'elle n'est pas peut aussi se révéler efficace et pertinent. L'objet ici, on l'a déjà souligné, n'est pas de fournir une approche exhaustive de la musique, mais d'en dégager des caractéristiques et des aspects opératoires dans une démarche géographique.

On postule ainsi plusieurs éléments :

- L'universalité. La musique est présente dans toutes les sociétés, et semble-t-il dans tous les groupes humains. Elle peut toucher d'une manière ou d'une autre tous les individus. En cela, elle touche à l'universel. Il devrait donc être possible de comparer ou d'étudier simultanément plusieurs pays / régions / sociétés et leur rapport aux musiques, les leurs et celles des autres.

- La diversité, la pluralité des formes et des utilisations. C'est une évidence de dire que la musique revêt des formes innombrables ; l'universalité n'est pas synonyme d'uniformité et la variété des styles est à peu près aussi importante que la variété des sociétés et des individus. Dans la mesure où toute combinaison consciente de sons et de silences peut prétendre au statut de musique, la diversité formelle est extrême et elle se double d'une diversité de l'utilisation et de la réception de la musique. D'une écoute distraite et passive à une pratique codifiée et ritualisée, la musique compose là encore un large éventail, dont l'existence même montre l'importance du rôle et de la place de l'objet et du phénomène «musique» au sein de la plupart des sociétés.

5 Un premier couple de notions se dégage : universalité / diversité. Dans le cadre d'une réflexion sur la mondialisation et sur la culture mondialisée, ce couple permet de réfléchir à l'objet musique en tant que phénomène à échelle planétaire, tout en interrogeant les modalités de cette universalité, et voir comment se négocie le passage à l'échelle locale.

- L'immatérialité : en tant que telle, la musique est un élément immatériel. Cette immatérialité est paradoxale. Pendant longtemps, elle a cantonné la musique à une pratique et à une écoute simultanées : la diffusion de la musique en était donc ralentie, et se faisait sur des étendues réduites. Mais cette immatérialité peut faciliter la circulation musicale, dans la mesure où elle permet de transcender toutes les échelles, de l'individu au monde, ainsi que les frontières, matérielles et symboliques. La musique, donc, ne se voit ni ne se touche, mais elle peut circuler.

- La mise en support. Au $\mathrm{Xx}^{\mathrm{e}}$ siècle, les innovations technologiques ont permis peu à peu de capturer les sons et de les reproduire sur des supports de plus en plus perfectionnés et de plus en plus légers. Du phonographe au fichier MP3, l'évolution technique a rendu la musique de plus en plus facilement accessible, pour un auditoire de plus en plus nombreux. Cette mise en support constitue probablement la rupture la plus importante de l'histoire de la musique, dans la mesure où elle a permis de matérialiser et de circonscrire le son et la musique. C'est un changement extrêmement important puisque, avant cette innovation technique, la musique ne pouvait être entendue que dans un contexte où sa production, sa diffusion et sa réception étaient simultanées : tout le processus était concentré dans un même espace et dans un même moment. La mise en support a permis d'élargir de manière exponentielle les champs de diffusion potentielle de la musique. Dès lors, sa diffusion, sa circulation et sa réception à toutes les échelles ont été révolutionnées par cette matérialisation.

6 Le couple immatérialité/mise en support dégage la notion de circulation musicale : la musique est un objet mobile et mouvant. Cette mobilité constitue un concept 
particulièrement opératoire dans la réflexion sur le lien entre musique et espace. Mais la circulation musicale est paradoxale, dans la mesure où, quelle que soit l'échelle de diffusion, du micro au macro, elle nécessite la présence d'au moins un individu pour être effective: le phénomène musique ne semble pouvoir exister que dans une dialectique de la diffusion et de la réception et la création musicale ne prendre son sens que dans la mesure où il $\mathrm{y}$ a au moins un individu pour la recevoir. Réfléchir sur la musique et son mode de circulation impliquerait donc nécessairement une réflexion sur les acteurs de cette circulation : à travers le prisme musical, il semblerait que la question de l'espace ne peut se concevoir qu'en lien avec celle de l'humain.

- L'inutilité. La musique, surtout depuis l'avènement de la "société de loisirs", est communément considérée comme quelque chose d'omniprésent mais de paradoxalement inutile. Qu'elle relève des domaines de la culture, de l'esthétisme, du religieux, du politique ou des loisirs, elle n'apparaît pas comme un besoin vital, et elle reste cantonnée au domaine de l'ornementation sonore. Significativement, le statut des musiciens est souvent ambigu et relève du même paradoxe, qui consiste à leur donner à la fois beaucoup de visibilité et peu de crédibilité. La musique, en tant que phénomène social, ne serait pas quelque chose de « sérieux.»

- La rentabilité et la valeur marchande. Cependant, cette inutilité se double d'un poids économique et marchand très important. Même associée uniquement à la sphère des loisirs et du temps libre, la musique représente aujourd'hui beaucoup d'argent; elle possède une valeur objective, et peu de secteurs économiques sont actuellement plus rentables et génèrent plus de capitaux qu'elle. On présuppose que ce poids économique est lié à la révolution technologique évoquée plus haut. En permettant à toute musique de se diffuser toujours plus largement et d'atteindre toujours plus d'auditoire, les supports techniques en ont fait un bien de consommation désirable et désiré, intéressant en lui-même mais aussi par sa capacité à devenir vecteur de discours, de modèles et de représentations.

7 Ce troisième couple de notions, tout en soulignant une fois de plus un des paradoxes de l'objet musique, permet de montrer à quel point la musique peut être quelque chose de matérialisé. Dans une réflexion géographique, cela permet de questionner son emprise spatiale, réelle comme idéelle, et par là même, d'interroger la notion de «territoire de la musique».

\subsection{Les sciences sociales et la musique}

\subsubsection{Anthropologie, sociologie... la vision « classique » de la musique}

8 La musique et le champ musical ont et sont toujours avant tout le champ privilégié des sciences humaines et des sciences sociales, dans une approche pluridisciplinaire au sein de laquelle la géographie ne tient pourtant qu'une place extrêmement restreinte.

En tant qu'objet à part entière, la musique est d'abord l'objet de la musicologie, qui consiste d'une part à étudier l'histoire et l'évolution des musiques - l'approche est avant tout une approche temporelle, même si elle ne peut totalement occulter le spatial ; d'autre part, la musicologie définit l'étude en détail d'œuvres musicales, qui, à l'instar des œuvres littéraires, sont étudiées à l'aune de leur mélodie, de leur technique, de leurs influences, etc. Mais, à compter du début du $\mathrm{xx}^{\mathrm{e}}$ siècle, dans un contexte d'exploration et de description encyclopédique du monde, la musique sous certaines 
formes - notamment sous celle des rites sociaux dans les sociétés dites traditionnelles a été approchée et appropriée par l'anthropologie, l'ethnographie puis la sociologie. L'étude de la musique s'est par conséquent soumise précocement aux jeux du brassage disciplinaire.

Jusqu'aux années 1950-1960, l'étude de la musique, en dehors de la musicologie, s'est beaucoup cantonnée à une approche anthropologique et ethnographique «traditionnelle », c'est-à-dire celle qui se place dans la lignée, côté anglophone d'un Evan-Pritchard ou d'un Malinovski, ou, côté francophone d'un Lévi-Strauss. Elle n'est pas étudiée comme objet à part entière, mais comme manifestation sonore de structures sociales et identitaires. Surtout, l'étude de la musique reste globalement cantonnée à un lointain géographique et, dans une certaine mesure, temporelle (l'étude du folklore occidental ayant aussi sa place dans cette approche, mais dans une moindre mesure). La musique des sociétés contemporaines, comme objet quotidien soumis autant aux évolutions techniques qu'aux héritages du passé, ne constitue pas un objet d'étude particulièrement fédérateur.

11 Ce n'est que dans les années 1960, avec l'École de Francfort et autour de la discipline encore nouvelle de l'ethnomusicologie, que la «construction sociale, politique et économique du fait musical » commence à être prise en compte, par Adorno et Benjamin, dans une double perspective de naissance des industries culturelles et de critique marxiste de la société. Ces premiers travaux constituent les jalons d'une tendance qui est allée croissant dans la recherche en sciences sociales jusqu'à aujourd'hui, et qui consiste à considérer la musique "en tant que culture » et "non pas comme objet dans la culture » (Guiu, $2006: 8-9$ ). Cette tendance a contribué à faire un peu plus de place dans l'étude de la musique aux approches et à la prise en compte de l'espace et des lieux propres à la géographie.

12 Par conséquent, depuis l'accélération des phénomènes de mondialisation, durant les dernières décennies, il y a eu dans les sciences sociales un brassage disciplinaire : la musique et le fait sonore sont, devenus un objet pour l'histoire (comme l'a montré A. Corbin en 1994 en bâtissant une histoire de la France du XIX ${ }^{\mathrm{e}}$ siècle autour des paysages sonores et des sens en général $\left.{ }^{1}\right)$ ) et non plus uniquement pour la musicologie et l'anthropologie. A cet élargissement à d'autres disciplines s'est ajouté un brassage thématique : « l'ensemble des sciences sociales s'intéresse donc à l'espace sonore et aux liens entre identité, territoire et musique. » (Guiu, ibidem) On a vu ainsi se généraliser dans les études d'anthropologie ou de sociologie urbaines l'emprunt de termes et de problématiques territoriales issus de la géographie, tandis que la géographie s'est pour sa part intéressée aux approches d'autres sciences humaines (ce que je développe dans le point suivant.)

13 Pour prendre un seul exemple, lié à l'objet de ce mémoire, l'émergence dans la deuxième moitié $\mathrm{du} \mathrm{xx}^{\mathrm{e}}$ siècle de musiques nouvelles, liées à la place inédite prise par la jeunesse dans les sociétés contemporaines, aux innovations techniques qui permettent d'amplifier la musique, et à la naissance de cultures décrites comme spécifiquement urbaines, a incité les anthropologues et les sociologues à réfléchir à la ville, à la diffusion et à la circulation mondialisée des cultures et des modes, à la revendication territoriale etc. Ainsi lorsqu'en 2003 une sociologue dirige un ouvrage collectif, en collaboration avec la Cité de la Musique de Paris, sur des musiques comme le rap ou la techno, elle ancre sa réflexion dans une dimension spatiale. L'ouvrage est ainsi intitulé Territoires de musiques et cultures urbaines, et le sous-titre précise : Rock, rap, techno... 
l'émergence de la création musicale à l'heure de la mondialisation (Laffanour, 2003). Le chapitre introductif ${ }^{2}$ évoque les notions de frontières, de limites, et la circulation des musiques sur la « sono mondiale »; la ville, comme espace public ouvert à la création ; les espaces de représentation et d'expression; les territoires artistiques; l'action enfin des pouvoirs publics sur certains espaces... autant de pistes de réflexion qui montrent la porosité, dès que l'on s'intéresse à la musique, des limites qui séparent encore la géographie de la sociologie et de l'anthropologie. Le choix de mon objet d'étude, qui porte non seulement sur la musique, mais sur la musique urbaine et actuelle, dans un contexte de mondialisation culturelle et économique, s'inscrit totalement dans cette nouvelle donne, à savoir des limites de plus en plus floues entre les différentes sciences humaines.

\subsubsection{Quelle place pour la musique en géographie?}

14 Dès lors, il est facile de se demander quelle est la place, actuellement, de la musique au sein de la géographie, voire d'en interroger la légitimité : si les approches sociologiques ou anthropologiques ont intégré à leur démarche des concepts qui étaient ceux de la géographie, le phénomène inverse s'est-il produit, et sous quelle forme? Mais, dans ce cas, qu'est-ce qui sépare encore les disciplines les unes des autres? En quoi la démarche du géographe peut-elle stimuler une réflexion singulière sur la musique?

15 Je me suis au départ surtout appuyée sur le chapitre de J. Lévy dans Le Tournant géographique consacré à la musique comme nouveau « territoire de l'improbable » (293); la réflexion y est centrée sur la musique "savante " plus que sur la musique issue de la culture populaire de masse, elle ne dépasse pas 1945 dans son cadre temporel et elle reste focalisée sur la musique européenne. Cependant, il introduit des champs de questionnements qui ont largement sous-tendu ma propre réflexion. La circulation des courants musicaux est ainsi mise en avant, puisque Lévy postule que, s'agissant des objets esthétiques, elle est importante, dense et précoce, par rapport par exemple aux objets techniques ou économiques. Il réfléchit aussi à la différence entre échanges culturels et échanges marchants: "la diversité des sous-espaces, souvent nationaux, résiste souvent davantage en art que dans le domaine des échanges marchands passant par la monnaie. » (296) Il inscrit enfin dans le même champ d'analyse la musique, les structures de pouvoir - notamment les États - l'univers urbain en ce qu'il peut favoriser les innovations, et le fait économique. Ce sont des axes de recherche que j'ai souvent repris au cours de ce travail.

16 Mais J. Lévy, quand il écrit cet article en 1999 s'inscrit dans une dynamique de renouvellement de la géographie, notamment de la géographie française, qui a eu lieu à partir de la fin des années 1970. S'agissant de la musique en elle-même comme objet géographique, la géographie anglophone a été le précurseur principal, comme le souligne R. Hudson (2006) dans une synthèse sur les études effectuées en terrain anglophone sur les liens entre musique, identité et lieux. Je reprends ici brièvement l'article de C. Guiu, cité déjà plus haut, qui dresse un état des lieux synthétique et dense de la géographie en musique :

17 La musique en géographie, "terrain défriché et dispersé» (p. 9) est un objet de recherche géographique valorisé par un certain nombre de chercheurs depuis une vingtaine d'années, qui dénoncent le primat du visuel et du paysager qui existe au sein de la géographie. On peut distinguer trois grandes périodes dans l'évolution de la 
thématique en géographie : les années 1970 comme point de départ significatif, la fin des années 1970 et les années 1980 avec l'accent mis sur les soundscapes enfin les années 1990 et les approches culturelles et postmodernes. Parallèlement, les pôles de recherche se sont aussi multipliés : des États-Unis, précurseurs, le champ s'est élargi à la Grande-Bretagne puis à la France, avant de faire des émules en Océanie (Australie et Nouvelle-Zélande.) Il y a donc eu un élargissement sans que pour autant il y ait beaucoup de dialogues entre les différentes aires de recherche: à l'instar d'autres objets et thématiques, les recherches francophone et anglophone restent très étanches l'une à l'autre. Entre 1968 et 2006, soit en presque 30 ans, C. Guiu dénombre 140 travaux de recherches géographiques, - dans le monde - portant sur le fait sonore. Mais c'est surtout depuis 2000 que le thème connaît une croissance significative, ${ }^{3}$ et que de plus en plus de chercheurs l'intègrent d'une manière ou d'une autre à leurs analyses.

À partir des années 1970, autour de George Carney, se met en place une "géomusicologie» dite de l'École de Berkeley: elle cultive une approche dite diffusioniste de la musique, proposant des cartes définissant des aires et des limites culturelles, et fondée sur des méthodes plus quantitatives que qualitatives. Cette approche est critiquée car trop matérialiste, trop descriptive, et trop orientée dans ses zones d'études (les musiques des minorités, noires ou amérindiennes, étaient ainsi totalement ignorées au profit de la country par exemple). Ce pendant a été corrigé par une approche plus sensible et sensorielle autour de Lowenthal, plus "axée sur les représentations, l'imagination et l'expérience sensible » (14) tandis que la notion de paysage sonore - soundscape - était développée et faisait des émules, dans les années 1980, et que l'étude des sources artistiques, à travers les paroles et les thèmes musicaux, devenait une source à part entière. "L'écoute sonore de l'urbain et du quotidien ", initiée dès 1971 par l'université de Vancouver avec le World Soundscape Project de R. Schafer s'intéresse non seulement au fait musical, mais aussi à tout ce qui peut constituer le pendant sonore d'un paysage, qu'il fasse sens ou non au sein d'un groupe humain. $\mathrm{Ce}$ travail «d'écologie acoustique » est repris alors par l'ethnographie (J.-F. Agoyard) ou par l'histoire (A. Corbin) dans les années 1980 : c'est à partir de cette période que l'on commence à voir une grande proximité dans les approches sur la musique par les différentes sciences humaines. Dans les années 1990, avec le «tournant culturel » qui a lieu en géographie, impulsé par la cultural geography anglophone, cette proximité ne fait que s'accentuer: "le "tournant culturel" est corrélatif des dynamiques de la postmodernité et révèle les changements sociaux des relations espace-temps. » (16) Du côté de la géographie française, où la recherche sur les faits culturels a connu un renouveau sous l'impulsion de P. Claval, de nouvelles thématiques se développent, notamment une géographie des pratiques sociales - sport, festivals, tourisme... - au sein de laquelle on conçoit parfaitement que la musique trouve sa place. Dans un monde où la globalisation favorise la réduction des distances et une déterritorialisation de plus en plus forte, la musique, en tant qu'objet immatériel mais circulant facilement, apparaît comme un objet d'étude pertinent et éclairant. Trois grands pôles de recherches sur la musique peuvent ainsi être dégagés dans les études qui en ont été faites depuis les années 1990 jusqu'à aujourd'hui: les musiques analysées comme vecteurs d'images et de développement, comme "puissant instrument de création d'une imagerie territoriale » de "stéréotypes territoriaux» et comme élément performatif influent un territoire. Le deuxième pôle est celui du lien entre musique et pouvoir, que celui-ci soit politique ou économique: outre le fait que la musique est classiquement un «instrument de légitimation, d'affirmation ou de contestation du pouvoir» (19), la musique permet aussi 
d'analyser les jeux de relations complexes entre territoires locaux et réseaux mondiaux, comme élément de renforcement ou de déstabilisation de communautés locales etc. Enfin, le troisième pôle est celui de l'émergence, autour et par certaines musiques, de nouveaux lieux pour de nouvelles «communautés imaginées »: le lien entre musique et identité est là encore mis en valeur, sans exclusive cependant.

C. Guiu conclut sa synthèse en montrant que, s'il y a "autant de géographies de la musique qu'il y a de géographies », la tendance récente est cependant «l'émergence de la notion d'embodiement et de gender dans l'étude des relations entre musiques, territoires et identités", alors que les analyses quantitatives, depuis les travaux de Carney, sont relativement délaissées. D'autre part, il existe des constantes dans toutes les approches récentes de la géographie: l'idée notamment que la musique est un indicateur «des sentiments d'appartenance, des mobilités, des valeurs et des comportements sociaux» et qu'elle dispose d'une valeur performative sur les dynamiques territoriales.

20 Les perspectives de la musique en géographie sont donc nombreuses, côté anglophone comme côté francophone. L'organisation en 2006 et en 2007 de deux journées scientifiques (qui ont suscité l'article que je viens de résumer) interrogeant les chercheurs toutes disciplines confondues sur l'intérêt d'une approche géographique de la musique montre qu'il s'agit d'une thématique qui gagne actuellement en visibilité. Les diverses communications ont montré à la fois la réelle pluridisciplinarité du sujet, mais aussi une récurrence de certains thèmes : le rap, en particulier, a fait l'objet de plusieurs communications, et la ville de la Nouvelle Orléans apparaît aussi comme un pôle particulièrement attractif pour les réflexions de tous types sur le lien entre la musique et le territoire.

21 Le travail que je présente ici est redevable de cette double évolution : conjonction entre différentes disciplines sur certains thèmes et sur certaines méthodes, et définition d'un thème qui doit beaucoup à l'introduction au sein de la géographie de thèmes « culturels. »

\subsection{Deux types d'outils de recherche}

\subsubsection{Expérience sur place}

Le travail présenté ici est basé sur deux périodes consécutives passées à Dar es Salaam en 2006 et 2007, dans des contextes différents. En 2006, j'ai effectué un stage de sept mois à la chancellerie diplomatique de Tanzanie, et je suis revenue pour cinq semaines en 2007, cette fois-ci dans le cadre du master, et dans une optique de recherche proprement dite.

23 Mon objectif quand j'ai effectué mon stage était de me familiariser avec la Tanzanie et la ville de Dar, déjà dans l'idée d'y effectuer un éventuel travail de recherche ultérieur, c'est pourquoi il me semble intéressant de l'évoquer brièvement. Je travaillais à l'ambassade en tant " qu'attachée politique ", ce qui consistait en fait pour l'essentiel à suivre l'actualité politique via la presse, et dans une moindre mesure, économique, de la Tanzanie; l'autre partie du travail visait à maintenir un contact avec les organisations internationales de développement, très présentes en Tanzanie et auxquelles la France contribue largement, ainsi qu'à assurer le suivi des relations entre le Quai d'Orsay et le TPIR (Tribunal Pénal International pour le Rwanda). D'un point de 
vue strictement méthodologique, un stage dans un petit poste diplomatique comme celui-ci présente de nombreux avantages ; le principal est qu'il m'a permis, sur le seul atout de ma position à l'ambassade, d'avoir accès à de nombreuses institutions importantes, et à rencontrer un certain nombre de personnes qu'il m'aurait été plus difficile et plus long de rencontrer autrement. D'autre part, le travail de synthèse qui m'était constamment demandé m'a effectivement permis d'acquérir une vision globale du pays : la multiplicité des sujets abordés m'a fait me pencher sur des thèmes aussi variés que les élections présidentielles à Pemba (Zanzibar), la place des femmes en politiques, les conflits d'intérêts entre petits mineurs et grandes compagnies minières, les réactions à « l'affaire des caricatures » de mars 2006... tous ces événements ne sont certes pas en prise directe avec mon sujet de recherche, mais ils m'ont néanmoins permis d'élaborer, par petites touches, un tableau de la Tanzanie de 2007. En termes de réseau et de vue d'ensemble, on peut donc dire que ce stage s'est avéré productif. Cependant, la méthode a aussi ses limites. Une des plus importantes à mes yeux reste le fait que le travail effectué ne m'a permis d'avoir qu'une vue très partielle et très biaisée $\mathrm{du}$ terrain. La majeure partie du travail s'est faite à travers les seuls filtres du rayonnement de la France, d'une part, et de l'idéologie du développement telle qu'elle est élaborée par les bailleurs de fonds internationaux d'autre part : cela donne parfois une vision finalement assez éloignée de la réalité. Le travail à partir uniquement de la presse anglophone est aussi limité : bien que la liberté d'expression soit une valeur plutôt respectée en Tanzanie, le contenu des journaux reste très inféodé à la ligne du gouvernement pour tout ce qui relève strictement de la politique, y compris dans les titres privés les plus 'indépendants' ${ }^{4}$ et l'actualité internationale est en général une reprise mot pour mot des dépêches des agences internationales. Quant aux sujets de société, à quelques exceptions près, ils sont souvent très éloignés de la vie quotidienne de la majeure partie de la population et semblent s'adresser à une classe moyenne occidentalisée, (voire américanisée), parfaitement anglophone et urbanisée dont on se demande si elle existe vraiment en Tanzanie ${ }^{5}$. Ils véhiculent donc un certain type de discours, de mise en scène du pouvoir et de représentations sur le pays qui ne sont pas en soi inintéressantes, mais qui ne peuvent en aucun cas constituer la seule source légitime à un travail sur un sujet comme la musique populaire. En l'occurrence, sur le Bongo Flava, les journaux anglophones se révèlent la plupart du temps très conservateurs (ne faisant que répéter le discours officiel en rigueur depuis l'Indépendance sur les musiques nouvelles) et complètement ignorants des évolutions de la musique et de la vie culturelle underground de Dar; si l'on excepte le cas du Gospel, et quelques lancements officiels d'albums financés par des sponsors importants ou des coopérations culturelles, la représentation de cette musique reste assez caricaturale et moralisatrice. C'est du côté de la presse en swahili qu'il faut se tourner si l'on veut des renseignements sur ce qui se passe ndani ya Bongo (au cœur de Bongo [Dar es Salaam]); il existe une presse gouvernementale en swahili, mais elle est concurrencée par des dizaines de titres quotidiens, hebdomadaires et bihebdomadaires ${ }^{6}$ qui donnent un aperçu probablement beaucoup plus fiable des réalités et des évolutions en profondeur de la société tanzanienne que leurs homologues anglophones. Les sujets internationaux sont beaucoup moins évoqués, à l'exception du football et des potins, au profit de thématiques plus divertissantes et plus légères : la musique, le sport, la mode, les romans-photos... Mais, à l'exception des titres les plus " sérieux », la lecture de ces journaux exige une connaissance assez fine du swahili, que 
je ne possède pas, ce qui m'a probablement privée d'un point de vue différent, via la presse, sur la société tanzanienne.

Néanmoins, cette expérience de plusieurs mois, sans être déjà orientée un travail de recherche précis, m'a quand même été très utile pour revenir en Tanzanie quelques mois plus tard. Ne pouvant rester sur place que sur une courte période, le fait de déjà bien connaitre les lieux, de parler un peu swahili et d'avoir un certain nombre de contacts sur place m'a fait gagner beaucoup de temps, matériellement et scientifiquement. Je n'aurais autrement pas pu revenir sur un temps aussi court. Revenue à Dar en février 2007, j'ai effectué un court travail de terrain, orienté cette fois-ci sur le monde de la production musicale, notamment de Bongo Flava, et surtout destiné à prendre des contacts et à rencontrer des gens d'un milieu que, pour le coup, mon expérience précédente ne m'avait que peu amenée à côtoyer. Cela s'est traduit par un petit nombre d'entretiens plus ou moins long avec des musiciens, par des recherches à l'université de Dar es Salaam (un mémoire notamment, celui de P. Magesho, était particulièrement utile) où la quasi-totalité des travaux de recherche ne sont accessibles que sur place, et des prises de contacts avec des journalistes.

Enfin, l'Unesco, qui oriente actuellement un de ses champs d'action sur l'industrie de la musique et les problèmes des droits d'auteur, s'était également montrée intéressée par mon projet de recherche; j'ai pu avoir aussi un entretien avec le responsable de la partie "Culture", qui a insisté sur le besoin actuel d'avoir une représentation cartographique des phénomènes musicaux dans la ville, que cela traduise les quartiers de création musicale, les zones de vente ou les zones de production. Selon lui, cette cartographie est une des conditions nécessaires à l'élaboration de plans d'action réellement efficaces sur le statut actuel des artistes... Cet entretien, même s'il ne pas m'a concrètement apporté d'aide en termes de terrain, s'est quand même révélé précieux car il montrait que l'objet que j'avais choisi et l'approche mi-culturelle miéconomique que je tentais de mettre en place étaient «dans l'air du temps. » À défaut de faisabilité, cet entretien a donc apporté une forme de légitimité extérieure à ma démarche.

\subsubsection{Internet, un outil de recherche à part entière}

Le fait de m'être intéressée dès mon travail de maîtrise à des problématiques de réseaux et de connectivité m'a conduite à me pencher sur le réseau par excellence que constitue Internet. Dans une société mondiale de plus en plus "en réseaux» (M. Castells, 1998) Internet pose question en ce qu'il représente de potentiels de connaissance, de canal de circulation culturelle et informative, et de capacité de mise en contacts d'espaces jusque-là très éloignés, aboutissant ainsi à ce qui s'apparente à une réduction de l'espace réel. D'autre part, au même titre que la musique, Internet présente un paradoxe, dans la mesure où il constitue un espace totalement virtuel, mais qui entraîne des modifications conséquentes et sans précédent sur la réalité.

La Toile tenait donc une large place dans mes hypothèses de travail. Je supposais qu'elle constituait, dans le cas de la musique tanzanienne, un outil important de réception et de diffusion. Théoriquement, elle doit en effet permettre la circulation de nouveaux morceaux, via le téléchargement et la compression du son en formats de fichiers de plus en plus légers, donc de plus en plus transportables; la circulation de nouvelles modes et de nouveaux courants musicaux, pas nécessairement disponibles autrement ; 
mais aussi la circulation de noms, et création de réseaux virtuels d'artistes ou de producteurs de musique disséminés à travers le monde.

D'autre part, la nature de mon objet de recherche m'a aussi poussée à utiliser de manière intensive les ressources disponibles via Internet. La bibliographie sur la musique tanzanienne est, à la base, peu abondante, et elle l'est encore moins en français ; d'autre part, l'objet en lui-même est en évolution constante. Les musiques et, plus globalement, les cultures urbaines changent extrêmement rapidement: si les logiques et les facteurs de changement évoluent plus lentement, en termes de contenu, ce qui était vrai au début des années 2000 ne l'est plus forcément en 2007. Cette rapidité est d'autant plus visible à Dar es Salaam, qui connaît actuellement des évolutions parfois tellement rapides qu'il devient difficile simplement d'en rendre compte. Dans ce contexte, Internet se présente comme le seul outil qui permette une mise à jour de tous ces changements : s'il y a peu de documentation écrite en format papier sur la musique actuelle tanzanienne, il y a au contraire pléthore de sites et de ressources numérisées disponibles sur Internet. Sans remplacer une étude sur le terrain, Internet permet d'avoir un aperçu du terrain en question et de se tenir au courant de ce qui s'y passe ; en cela, il y a bien un phénomène de réduction de l'espace qui peut être intéressant à exploiter au cours d'une recherche.

\section{Internet en Tanzanie}

Quand on dresse le tableau du rôle et de la place d'Internet en Tanzanie et, dans une moindre mesure, au Kenya, le bilan reste cependant assez mitigé, surtout si l'on compare avec l'Afrique de l'Ouest, par exemple le Sénégal et le Nigeria, où il fait désormais partie du quotidien d'une très large proportion de la population, au moins urbaine. On l'a évoqué en introduction, cela s'explique en partie par le fait que cette zone de l'Océan Indien n'est pas encore reliée aux câbles sous-marins mondiaux, ce qui entraîne une faible capacité du réseau actuel. Les choses devraient changer en 2008, date à laquelle cette connexion devrait être faite : il sera alors intéressant de voir quels seront les impacts de cette installation.

En attendant, l'accès à Internet continue en Tanzanie comme au Kenya à être un puissant indicateur d'inégalités sociales et territoriales. Le clivage villes-campagnes est comme il se doit encore plus fort que ceux qui existent déjà en matière de distribution d'électricité. Mais ces inégalités se jouent aussi entre villes de différentes tailles et de différentes attractivités, à l'intérieur des villes mêmes, et aussi entre utilisateurs occasionnels, clients de cybercafés, et utilisateurs privés, disposant de leur connexion à domicile. Ainsi, les villes de Dar es Salaam et Nairobi disposent sans surprise des meilleures connexions; au Kenya, Mombasa dispose également de bonnes infrastructures. À ce stade, le niveau d'accès à Internet n'est que le reflet d'autres types de répartitions d'infrastructures et d'équipement. Côté tanzanien, les villes du Kilimandjaro (Arusha et Moshi) ainsi que Zanzibar sont également plutôt bien connectées : le dynamisme touristique de ces espaces peut en grande partie expliquer cela, étant donné qu'ils attirent chaque année une population de touristes de plus en plus nombreuse (environ 500000 touristes en Tanzanie pour 2006), avec un fort pouvoir d'achat, et ayant depuis longtemps intégré Internet dans ses pratiques quotidiennes. À échelle plus fine, sans surprise, c'est dans les quartiers les plus centraux et les plus actifs que l'on trouve le plus de cybercafés et autres connexions à l'intention de cette population particulière. 
31 À Dar, même si les logiques sont peu ou prou les mêmes, les zones où l'accès Internet est possible sont plus nombreuses et plus dispersées. Si l'on tient aux cybercafés, c'est dans les quartiers centraux de Kariakoo, Upanga et de Kivukoni Front, tous denses, actifs et bien équipés, que l'on trouve le plus de connexions ; les quelques grands malls répartis dans la ville sont eux aussi souvent bien pourvus. Enfin, les quartiers relativement proches du centre-ville, où se mêlent populations pauvres, moyennes et aisées, à l'instar de Mikocheni $\mathrm{A}$ et $\mathrm{B}$, ou de Kinondoni, disposent également d'un certain nombre de cybercafés. En revanche, ceux-ci sont quasiment inexistants dans les quartiers les plus huppés de Msasani, Masaki et Oyster Bay: Internet y est présent, mais quasiment exclusivement chez des particuliers qui disposent de leur propre ligne.

Quoi qu'il en soit, il est frappant de constater combien ce réseau de cybercafés et de points d'accès à Internet est faible par rapport à l'étendue de l'agglomération. Il évite déjà toutes les zones non électrifiées, nombreuses dans cette ville où l'urbanisme a pendant longtemps été inexistant, et, même dans les quartiers bien équipés, il reste assez anecdotique. (Voir carte $n^{\circ} 2$ en annexe sur Internet.) En comparaison par exemple avec Dakar, l'accès à Internet des citadins de Dar es Salaam est très médiocre, et il ne fait guère partie des pratiques quotidiennes, mais il faut admettre aussi que la mauvaise qualité de la plupart des points d'accès, ainsi que leur cherté (environ 500 TSh l'heure, soit 35 centimes) est assez dissuasive.

\section{La sphère musicale et Internet}

33 À travers ce tableau édifiant, il ne serait donc guère surprenant de voir Internet complètement délaissé par le secteur musical. Pourtant, la situation est là encore paradoxale, dans la mesure où, malgré cette faiblesse relative, il y à Dar es Salaam et sur la vie musicale tanzanienne en général de nombreuses ressources en lignes, et un certain dynamisme.

On trouve en effet sur Internet un panel de magazines en ligne, réalisés à Dar es Salaam, le plus souvent en anglais et en swahili, qui donnent aux internautes sur un mode plus ou moins communautaire toutes les actualités culturelles et les événements à venir. Le plus connu et le plus généraliste est le site DarHotWire, qui se veut un magazine en ligne sur la Tanzanie, mais dont les sections consacrées au Bowgo Flava occupent une place conséquente. On peut aussi citer SwahiliRemix, Mahusiano Music, Ngome Entertainment... Tous sont plus ou moins centrés sur le Bowgo Flava, et se revendiquent de la culture jeune, urbaine et moderne qu'est censé véhiculer cette musique, tout en évoquant aussi, plus globalement, les musiques qui plaisent au public tanzanien, du taraab au rap nord-américain.

On peut prendre comme exemple le site Mahusiano $^{7}$ qui se présente sur sa page d'accueil comme "the land of bongo flava music ». Créé en septembre 2006 par un des fondateurs d'une compagnie alors naissante de conseils en investissements, MK Group, Mahusiano.com a pour vocation déclarée est de «faire connaître la musique tanzanienne au reste du monde » (« to introduce Tanzanian Music to other parts of the world»). Le site est presque exclusivement rédigé en swahili - contrairement par exemple à SwahiliRemix, dont les créateurs sont tanzaniens et européens, et qui revêt donc une dimension diasporique - : il est avant tout destiné à un public tanzanien, ou en tout cas originaire d'Afrique de l'Est. La présentation est classique, et divise sommairement le site en rubriques de vidéos, de fichiers audio, de photos et de forums de discussions. La partie 
intitulée "Mahusiano Audio Center" divise les chansons diffusées en cinq styles différents : Bongo Flava, Bongo Dance Music, Taarab, Zi/ipywdwa (littéralement : « ceux qui étaient aimés » c'est-à-dire les "bons vieux classiques ») et enfin les Nyimbo za Injili, « chansons de l'Évangile » à caractère religieux, dont le succès est grandissant en Tanzanie. Les actualités diffusées concernent essentiellement la vie musicale à Dar es Salaam: dates de concerts et de sorties d'albums surtout. Dans une moindre mesure, mais cependant relativement présentes, les nouvelles et les potins sur les stars de la musique afro-américaine occupent aussi une place non négligeable (à l'instar de la presse écrite, qui reprend souvent mot pour mot, en anglais et en swahili, les dépêches "people» des agences de presse internationales.) Enfin, comme la plupart des magazines en ligne, Mahusiano se veut interactif et participatif : une large place est faite aux forums, aux chats, et aux espaces d'expression pour les lecteurs. Les statistiques affichées sur le site montrent cependant que pour l'instant, il est resté dans la confidentialité : le 13 mars 2007, le site a atteint son plus haut pic de fréquentation avec... 13 utilisateurs en ligne simultanément, sur un total de 67 abonnés. L'échelle de diffusion mondiale n'est pas vraiment atteinte pour l'instant.

Parallèlement à ces sites généralistes, un petit nombre de producteurs de musiques se sont également mis à travailler avec Internet, depuis le début des années 2000. La mise en ligne de samples et de morceaux instrumentaux doit en effet permettre à des groupes très dispersés de travailler quand même ensemble : un certain nombre de producteurs et d'artistes passent en effet du temps, voire sont installés, à l'étranger, en particulier aux États-Unis et, dans une moindre mesure, au Royaume Uni. Les sites de ces producteurs permettent en théorie ces collaborations, notamment dans le milieu hiphop, où le haut degré de numérisation de la musique et le faible besoin d'instruments de musique facilitent un travail largement opéré grâce à l'informatique. Si l'on en croit Peter Magesho, dans sa thèse sur le Bongo Flava (2003), les principaux producteurs de rap ont mis en place leur site Internet pour favoriser ce type de création; écrite en 2003, il cite par exemple Poa Records, basé à Dar es Salaam, ou Grandmaster Records à Arusha. Ces sites existent toujours, mais sont plus ou moins régulièrement mis à jour et semblent peu fréquentés; quant à la dimension de collaboration en ligne évoquée par Magesho, elle n'est pas vraiment visible, tout au plus est-elle facilitée par la rubrique «Contacts » présente sur tous les sites.

Pour résumer, on peut dire que la relation entre Internet et le monde de la musique en Tanzanie est fondée sur une double logique. La première est une logique de diffusion, en essayant de mettre à profit de la manière la plus large possible le potentiel de diffusion infinie que devrait permettre le réseau; il semble que cette première stratégie ne soit que modérément couronnée de succès. En revanche, il existe aussi dans ce fonctionnement virtuel une vocation fortement communautaire, qui vise à maintenir des liens entre les artistes à l'étranger et ceux qui sont restés à Dar es Salaam. Il s'agit d'un petit réseau, composé tout au plus de quelques centaines de personnes, mais, ici, Internet remplit parfaitement son rôle; s'il ne parvient pas à susciter une création musicale basée sur les relations virtuelles, il contribue quand même à maintenir des liens vivaces au sein d'une communauté artistique restreinte, mais assez homogène, et répartie dans le monde entier. 


\section{Internet et MySpace : des outils de recherche nouveaux} potentiel qui, à l'usage, ne tient pas nécessairement ses promesses, mais il est néanmoins symbolique, et tend à évoluer depuis deux ou trois ans avec la montée en puissance du Web 2.0, ou Internet participatif. Le principe du Web 2.0 est celui d'un édifice en perpétuelle construction, où ce sont les internautes eux-mêmes qui font évoluer les sites qu'ils consultent. Les créateurs de ces sites n'ont en fait qu'à mettre en place des trames virtuelles, et c'est le public qui se charge du contenu, plus ou moins surveillé par des modérateurs. Les sites les plus emblématiques actuellement sont par exemple YouTube, site de vidéos en ligne, ou Wikipédia, encyclopédie multilingue où tout un chacun est susceptible d'apporter des modifications et des compléments aux articles qu'il lit. En termes d'Internet participatif, le réseau MySpace est également depuis sa création en 2003 un des sites qui a connu la plus grande notoriété. « MySpace est un réseau social, accessible à tous. Il suffit de s'y connecter pour se faire des "amis", selon ses affinités musicales, sportives, cinématographiques ou autres. » (Le Monde) ${ }^{8} \mathrm{Le}$ principe de base, simplissime, est le suivant : n'importe qui peut s'inscrire, ce qui donne l'accès à une page personnelle, qu'il est possible de transformer et de changer à sa guise. La structure du site permet d'insérer dans son profil des photos, de la musique, des vidéos... une utilisation un peu plus poussée permet de personnaliser aussi l'ensemble du style de la page, d'ajouter des liens vers d'autres sites, des blogs etc. La page ainsi élaborée constitue la vitrine de son propriétaire; il s'agit ensuite de se faire un maximum «d'amis » - d'où le slogan du site, «MySpace, a place for friends » - qui laisseront s'ils le veulent des commentaires sur un espace réservé à cet effet. Pour se constituer un réseau « d'amis », il faut envoyer une « invitation » sur la page souhaitée, ou y répondre. Le fait d'avoir quelqu'un parmi ses amis permet de voir tous ses amis à lui, et de se constituer par ces liens son propre réseau. Les amis ainsi rassemblés peuvent ensuite laisser s'ils le souhaitent un commentaire sur la page visitée... le nombre d'amis, de commentaires, et la notoriété des «amis » renforçant évidemment l'intérêt de la page : le site marche en fait autant à la découverte d'autrui qu'à l'ego des utilisateurs!

pace n'est pas le seul site à proposer ce genre de démarches : d'autres sites, comme Hi-Five ou Nettbuzz sont basés sur le même principe. Mais, par son fonctionnement extrêmement simple, il a connu un engouement rapide; s'il s'agit d'une plate-forme théoriquement généraliste, et sans autre but affiché que l'élaboration d'une communauté virtuelle9 MySpace a fondé sa réputation aussi sur la musique.

Les musiciens « ont été les premiers à saisir l'intérêt de ce site, qui leur offre un lien direct et permanent avec leurs fans. En faisant de leur profil un mini-site peÉsonnel et grâce au parrainage en vigueur sur MySpace, ces artistes ont fédéré autour d'eux des communautés très efficaces. » (Ibid.) Le hip-hop nord-américain a été précurseur en la matière : The Roots, groupe culte de Philadelphie qui expérimentait le concept depuis plusieurs années sur son site communautaire Okayplayer, a ainsi été un des premiers à faire de MySpace un lieu virtuel couru à la fois par les artistes et le public. Les créateurs du site ont suivi la tendance en mettant au point un modèle de page MySpace Music, qui permet aux musicÉens de mettre en ligne plusieurs morceaux et de se créer un site Internet miniature, et totalement gratuit. On mesure ainsi l'intérêt potentiel de ce genre de site pour des musiciens, d'autant plus s'ils ne sont pas très connus et s'ils ont peu de moyens : le fonctionnement en réseau permet parfois un effet «buzz» qui 
amène une notoriété soudaine et massive, et assure quoi qu'il en soit une diffusion d'information à moindres coûts qui explique le succès de MySpace, qui compte début 2007 plus de 140 millions de membres... ${ }^{10}$

41 C'est en analysant ce fonctionnement que j'ai tenté de me servir d'abord d'Internet, puis de MySpace, comme d'un outil de recherche à part entière : le principe était à la fois de me renseigner sur la scène actuelle est-africaine, notamment hip-hop, et à la fois de créer mon propre réseau virtuel «d'amis" en ciblant des musiciens ou des acteurs de la musique tanzanienne, et en essayant dans la mesure du possible de rentrer en contact avec eux.

J'avais commencé à me servir d'Internet comme simple spectatrice : comme n'importe quel auditeur un tant soit peu investi, j'ai commencé par écumer les sites musicaux, en me focalisant d'emblée sur les sites, nombreux et dynamiques, intéressés par les cultures urbaines sur le continent africain dans sa globalité ${ }^{11}$; cela m'a donné des indices sur la place de la scène tanzanienne dans le contexte africain, et permis de constater qu'il s'agit d'un pôle d'une certaine influence et d'une certaine notoriété, même s'il ne concurrence pas les géants ivoiriens et congolais pour la musique de danse, et sénégalais et sud-africain pour les musiques issues du hip-hop. Mon autre grande source de base a été la radio 1Xtra, radio anglaise faisant partie de la BBC, dédiée à la «new black music »; une fois par semaine, l'émission Destination Africa, présentée et organisée par le Kényan DJ Edu, fait le point sur toutes les nouveautés hiphop du continent africain dans son ensemble (Maghreb compris, ce qui est assez rare pour être souligné.) Certains artistes sont déjà très connus, mais d'autres ne sont pas du tout distribués en dehors de leur pays ou de leur région; en cela, l'émission constitue une mine de renseignements musicaux. C'est à partir de la liste des artistes diffusés que j'ai précisé mon champ de recherche; beaucoup de ces artistes n'ont pas de sites Internet, mais ont en revanche leur page personnelle sur MySpace. C'est ce qui m'a décidée à créer un profil, assez sommaire, mais où je précisais mon objet d'étude, et à l'utiliser comme un terrain de recherche - et plus seulement d'observation - virtuel, en envoyant un maximum « d'invitations. »

Même si je n'ai pas rencontré tous mes "amis", ma démarche s'est avérée utile puisqu'elle m'a permis de rentrer en contact avec deux musiciens importants en Tanzanie, FidQ et Dola Soul. FidQ est une des principales figures du rap tanzanien actuel ; basé à Dar es Salaam, il commence à atteindre une certaine notoriété au niveau africain, et se revendique comme un artiste hip-hop, et non Bongo Flava, même s'il est souvent présenté dans la presse tanzanienne comme l'un des chefs de file de ce style musical. Je l'ai rencontré plusieurs fois lors de mon passage à Dar es Salaam. Dola Soul a.k.a Balozi ${ }^{12}$ est l'un des pionniers du mouvement rap en Tanzanie; fils de diplomate, élevé longtemps à l'étranger, il a été membre dans les années 1990 d'un groupe appelé Dee-Plow-Matz (en référence à la profession des parents) qui a contribué à la diffusion du rap. Il termine aujourd'hui ses études à New York et s'engage activement pour la promotion du rap africain à l'étranger ; c'est d'ailleurs dans cette optique-là qu'il m'a écrit et m'a proposé son aide en me fournissant des noms de personnes à contacter et des numéros à appeler une fois arrivée à Dar es Salaam. Il a également longuement répondu par e-mail aux questions que je lui posais, de manière très construite et très intellectualisée. Je ne l'ai jamais rencontré au sens propre du terme, mais il s'est avéré être, concrètement, un contact des plus intéressants. 

amener Internet à une recherche sur des lieux réels, qu'à l'importance en termes de potentiel de diffusion qu'il pouvait avoir dans un domaine comme la musique. Il est évident qu'un terrain de recherche ne peut se cantonner à des recherches sur Internet. Il est évident aussi qu'Internet ne peut suffire à analyser et expliquer les phénomènes que je souhaite étudier, à savoir les liens entre mondialisation, diffusion et circulation des pratiques musicales, et changement des territoires urbains ; notamment parce que, comme le souligne P. Magesho, Internet ne touche encore en Tanzanie qu'une minorité de la population urbaine (elle-même encore minoritaire au niveau national.) Si on reprend le cas de MySpace, on constate qu'un groupe comme XPlastaz, qui est probablement le groupe tanzanien le plus connu, a reçu environ 11500 visites en deux ans et demi ; dans le même laps de temps, un rappeur de renommée internationale comme le New-Yorkais Talib Kweli a reçu 1620000 visiteurs sur sa page MySpace... Le décalage est finalement assez représentatif du décalage qui existe dans d'autres domaines entre le continent africain et le reste du monde. Il montre quoi qu'il en soit que surfer sur le web ne fait pas encore partie des pratiques culturelles quotidiennes en Tanzanie, et que, s'il représente une infinité de possibles, ceux-ci ne sont encore que des horizons lointains.

\section{NOTES}

1. CORBIN A. (1982). Le Miasme et la Jonquille : l'odorat et l'imaginaire social XVIII-XIX ${ }^{e s}$ siècles, Paris, Aubier Montaigne. 1994, Les Cloches de la terre. Paysage sonore et culture sensible dans les campagnes au XIX ${ }^{e}$ siècle. Paris, Albin Michel.

2. «Musique et territoire, faut-il choisir ? La sono mondiale se joue des limites. »

3. Par le biais notamment de numéros spéciaux de grandes revues internationales: Journal of Cultural Geography, 18/1 1998, Géojournal, 2006, vol. 65.

4. The Guardian, The Citizen, The African et The Daily News sont les principaux titres anglophones disponibles en Tanzanie. La presse en swahili est en revanche foisonnante.

5. Les sujets de société sont par exemple la crise d'adolescence, les régimes et les exercices à faire en salle de sport, la Saint-Valentin... Autant de sujets qui ne touchent 
qu'une infime partie de la classe moyenne tanzanienne, et qui ne touchent pas non plus la population expatriée à qui cette presse est aussi en grande partie destinée. Cependant, certains chroniqueurs écrivent sur des sujets beaucoup plus réalistes et quotidiens : par exemple la malchance de croiser un ami sans le sou dans un bar et de devoir lui offrir une bière, ou une cousine et ses amies dans un dala-dala, qui profiteront de l'aubaine pour voyager gratuitement.

6. Quelques titres: Nipashe!, (Dis-moi), Mwananchi (équivalent du Citizen), Burudani (Divertissement), BaabKubwa (journal consacré au Bongo Flavour).

7. www.mahusanio.com

8. DE PLAS O., "MySpace, un puissant outil de marketing sur Internet ", Le Monde, 08/01/2007.

9. Porte ouverte aussi à toutes les opportunités comme à toutes les dérives: les utilisateurs peuvent indiquer dans leur profil pourquoi ils sont inscrits : « Rencontres, Relations sérieuses, Amis, Réseau » [professionnel].

10. Le site a été racheté par Rupert Murdoch en 2005, qui l'estime aujourd'hui à 6 milliards de dollars, soit dix fois la valeur d'achat.

11. Citons Africultures, pour les francophones, Africanhiphop, Afropop, Afromix côté anglophone.

12. Balozi signifie "ambassadeur " en swahili, référence autant au métier de son père qu'à sa volonté de promouvoir le rap africain et une image positive de l'Afrique en général. Quant au nom Dola Soul, si Dola est un prénom swahili, le pseudonyme renvoie au groupe new yorkais De La Soul, dont les albums font désormais partie des classiques de la culture hip-hop.

INDEX

Index géographique : Tanzania| Tanzanie 